\title{
Development of a materials science course to serve diverse constituencies
}

\section{Dr. Melissa Eblen-Zayas, Carleton College}

Melissa Eblen-Zayas is an associate professor of physics in the Physics and Astronomy Department at Carleton College in Northfield, MN. She completed her BA in physics from Smith College in Northampton, MA and her PhD in physics at the University of Minnesota, Twin Cities. She an experimental condensed matter physicist interested in the electronic and magnetic properties of correlated electron materials as well as physics education. 


\title{
Development of a materials science course to serve diverse constituencies
}

\begin{abstract}
Seven years ago, the Carleton College physics department began offering an introductory materials science course for physics and chemistry students with interests in materials science and engineering (MSE). However, in order to appeal to a broader audience and provide more focus for a course that offered extensive breadth but limited depth, the course has evolved over the years to concentrate on materials science, energy, and the environment. The revised course begins with an overview of the relationship between material structure and properties, with a focus on the mechanical properties of materials, but then contains four units that consider applications of materials science to address environmental and energy issues. The four units are materials for solar energy conversion, smart materials and energy applications, materials for green buildings, and materials life cycle analysis.
\end{abstract}

This modified course aims to serve two groups of students: physics and chemistry majors with an interest in MSE and environmental studies majors. This paper provides an overview of how the course has evolved, including the learning goals, course content, and assessment tools, as well as possible future directions for this course. In addition, the paper discusses the successes and challenges in trying to address the needs and interests of students in these two different constituencies.

\section{Introduction}

At small liberal arts colleges, students interested in materials science and engineering (MSE) often major in physics or chemistry before pursuing graduate study in engineering. However, in any given year, the number of students interested in pursuing MSE is small, making it difficult to regularly offer an introductory materials science course that is directed only at this constituency. Since 2005, the physics department has offered an introduction to materials science course on a biennial basis. This course meets three times per week and does not include a lab. The first two times it was offered, this course provided a broad overview of the relationship between materials processing, structure, properties, and performance. More recently, the course has been changed to provide an introduction to materials science by considering select examples of how materials science might be relevant to addressing energy and environmental issues. This revised course aims to serve more diverse constituencies of students, including students interested in MSE, students interested in energy issues, and students in the environmental studies program. This integrated course offers unique opportunities to bring students with diverse background together to get a taste of materials science in the context of broader societal issues, but it also presents some unique challenges.

\section{Background and motivation}

At Carleton College, nearly two thirds of physics and chemistry majors go on to graduate study. Many students who major in the physical sciences choose to pursue graduate work in engineering fields, including materials science and engineering. In addition, the mission 
statement of the College states, "In their chosen fields of study, students strengthen their capabilities for disciplinary and interdisciplinary research.” Materials science is by nature interdisciplinary, and teaching a materials science course aimed at students with diverse scientific backgrounds helps foster interdisciplinary learning. It was with the goal of serving students interested in exploring the field of MSE and fostering interdisciplinary study in the sciences that Physics 260 Intro to Materials Science was introduced in the fall of 2005. In order to attract students with a breadth of scientific backgrounds, the only pre-requisites for this course were either introductory physics or introductory chemistry, with the completion of one more science class at the 200-level being strongly recommended. The class enrolled between 15 and 20 students, usually physics and chemistry majors, as well as a few geology and biology students. Nearly all students who enrolled had significant coursework in the physical sciences beyond the minimum prerequisites.

Although student response to the course was positive from the beginning, several trends converged that led to the modification of the materials science course. First, increasing numbers of science students expressed interest in energy and environmental issues. Second, in the 20092010 academic year, environmental studies (ENTS) changed from a concentration to a fullfledged interdisciplinary major at the College. To address these changes, in the winter of 2011, Physics 260 Intro to Materials Science was taught making extensive use of examples of how materials science might contribute to energy and environmental issues. The pre-requisites for the course remained the same, but now the expectation was that many students would not have taken any physics or chemistry courses beyond the introductory level. The hope was to attract a larger cross-section of students, including those students with interests or majors in environmental studies. The content of this revised course was significantly pared down, but sacrificing breadth allowed more in depth consideration of a few topics, namely materials for solar energy conversion, smart materials for energy efficiency, materials for green buildings, and materials life cycle analysis. In addition, the assessments of student learning, which previously relied heavily on problem sets and exams, were modified to focus more on conceptual understanding.

\section{Physics 260: Intro to Materials Science}

Learning objectives. In 2005, Physics 260 began as an effort to provide students with a taste of the vibrant, interdisciplinary, and growing field of materials science. In a small liberal arts college without any follow-up courses in MSE, we decided that the two overarching objectives for this course were to enable students to 1) assess whether a particular material is appropriate for a particular application through consideration of the structure, properties, and processing of a material, and 2) to research and evaluate a material and its appropriateness for potential applications and present their findings to a specified audience. We also wanted to use this course to develop ancillary skills in written communication, including communication of quantitative information.

Content. Originally, course content was selected primarily based on what is typically covered in an introductory materials science and engineering course that is offered at a large university. ${ }^{1}$ The course began with a unit exploring the structure of materials: types of bonding, crystal structure, defects, and disorder in crystalline materials. Emphasis was placed on similarities and differences between different classes of materials -- metals, ceramics, and polymers. The second 
unit focused on metals, including alloys and phase diagrams. The third unit focused on the polymer chemistry and properties, and particular processing approaches. The final three units focused on mechanical, electrical \& optical, and magnetic properties of materials.

Assessment. Because the course was aimed at physics and chemistry students who were potentially interested in graduate study, assessment of student learning primarily took the form of weekly problem sets and several exams. The goal was for students to be able to make quantitative calculations of materials structure and properties and then compare calculations to actual measured values. In this way, students had the opportunity to see how various models they were using matched with the real behavior of materials. To support ancillary skill development, students also did a number of writing assignments throughout the course that asked them to either apply what they had learned to new situations or to convey their knowledge to a general audience. For example, when learning about mechanical properties of materials, students were asked to choose one piece of sporting gear and explain to a layperson how mechanical properties of different materials impacted the sports user (i.e. what type of bicycle rider might prefer bicycle frames made of carbon, steel, aluminum, titanium, etc).

Student response. This course was taught twice, once in the 2005-2006 academic year and once in the 2007-2008 academic year. Student response on course evaluations was generally positive, with students who self-identified as having an interest in engineering noting that they appreciated the applied nature of the course. However, a number of students mentioned that they would have preferred to cover fewer topics in more depth. Because this course does not serve as a prerequisite for any other MSE courses at the College, we decided to revamp the course to focus on a few topics in greater depth (Table 1).

\begin{tabular}{|l|l|l|}
\hline & $\begin{array}{l}\text { PHYS 260: Intro to materials } \\
\text { science }\end{array}$ & $\begin{array}{l}\text { PHYS 260: Intro to materials } \\
\text { science (with environmental } \\
\text { examples) }\end{array}$ \\
\hline Unit 1 & $\begin{array}{l}\text { Materials structure: bonding, } \\
\text { crystal structure, defects, disorder }\end{array}$ & $\begin{array}{l}\text { Mechanical properties and } \\
\text { materials structure }\end{array}$ \\
\hline Unit 2 & Metals: Alloys/phase diagrams & $\begin{array}{l}\text { Materials for solar energy } \\
\text { conversion }\end{array}$ \\
\hline Unit 3 & Polymers & $\begin{array}{l}\text { Smart materials for energy } \\
\text { efficiency }\end{array}$ \\
\hline Unit 4 & Mechanical properties & Materials for green buildings \\
\hline Unit 5 & $\begin{array}{l}\text { Electrical properties \& optical } \\
\text { properties }\end{array}$ & $\begin{array}{l}\text { Plastics and materials life cycle } \\
\text { analysis }\end{array}$ \\
\hline Unit 6 & Magnetic properties & \\
\hline
\end{tabular}

Table 1: Comparison of the content of Physics 260 before and after course revision. 


\section{Phys 260: Intro to Materials Science (with environmental examples)}

Learning objectives. In the winter of 2011, the revised Physics 260 course was offered, with a commitment to include numerous examples of the relationship between materials structure, properties, and processing in the context of environmental and energy issues. This focus on the potential of MSE to make a difference in addressing real world problems and improving people's lives is consistent with the message in the 2008 National Academy of Engineering study Changing the Conversation: Messages for Improving Public Understanding of Engineering. ${ }^{2}$

The two overarching objectives for the revised course were only slightly modified from the previous version of the course. Namely, after taking the course students should be able to 1) assess whether a particular materials is appropriate for a particular application through consideration of its structure, properties, and processing, and 2) to research and evaluate how materials can be used to address environmental and energy challenges and communicate your analysis to an appropriate audience. The first goal is identical to the previous course. For the second goal, rather than asking the students to generically research materials and potential applications, students focused on analyzing how materials can be applied to address particular energy and environmental issues. Development of skills for written communication of quantitative information remained a priority.

Content. The revised course began by focusing on the structure, property, and processing relationships in the context of mechanical properties. After introducing concepts such as stressstrain diagrams, strength, and hardness, the class explored how the bonding and crystal structure of materials impacted the mechanical response of materials. Instead of introducing crystallographic notation in a stand-alone unit, the relevant concepts were introduced in the context of being able to calculate slip planes and slip directions. Similarly, the discussion about phase diagrams and metal alloys allowed many concepts to be discussed in the context of how materials structure and processing impacts the mechanical properties of materials. This approach provided a more cohesive consideration of a number of topics. In addition, historically, the development of materials was often driven by an interest in improving their mechanical properties. By providing a discussion of mechanical properties of materials and the historical impact of these developments at the beginning of the course, students begin to see how materials development can have a significant societal impact. ${ }^{3}$ This historical reflection on the societal impact of new materials paved the way for examining examples of how materials science and engineering today might be used to address some of society's environmental and energy challenges.

The remainder of the course was dedicated to considering several ways in which materials science and engineering can have an impact on energy and environmental challenges. The four applications that the course focused on were materials for solar energy conversion, smart materials for energy efficiency, materials for green buildings, and materials life cycle analysis. The modified course included many of the same topics, but they were taught in context of a particular application, using a just-in-time approach. ${ }^{4}$ For example, electrical properties of materials fit well in the unit on solar energy conversion, where exploration of band structure, semiconducting materials, and p-n junctions leads to learning about the operation of traditional Si photovoltaics (PV). 
Each of the four applied units in the modified course provided an opportunity to study fundamental properties of materials, as well as applications and current research directions. By organizing the course around four topics that are active research areas, graduate school bound students with more advanced physics and chemistry background were encouraged to read the primary literature to stretch themselves beyond what was covered in class. ${ }^{5}$ The unit on solar energy conversion included both photovoltaics and solar thermal power systems. As discussed already, traditional Si PV provided an excellent context in which to consider semiconductors and their applications. We modified some of the materials from the Modern Physics curriculum developed by the University of Colorado Physics Education Research group for students to learn about band structure and semiconductors. ${ }^{6}$ In addition to Si photovoltaics, the students also explored alternative PV devices, including dye-sensitized organic PV and quantum dot PV. The dye-sensitized PV introduces students to some electrochemistry principles, while the quantum dot PV introduces some of the concepts of simple quantum well systems. In the context of each of the technologies, students explore what materials are being used and what are the advantages and disadvantages of each type of PV technology. For solar-thermal power systems, materials considerations are important for selection of heat transfer fluids and energy storage medium. The second unit on smart materials for energy efficiency introduces students to the concept of smart materials, and then focuses on thermoelectric and piezoelectric materials for small-scale energy harvesting. Piezoelectrics highlight clearly the structure/property connections because deformation of the crystal structure due to mechanical force produces the polarization in these materials. Thermoelectrics allow students to explore the connections between thermal and electrical transport in materials. The third unit discusses materials for green buildings. We chose to focus on materials for windows and insulation, which have the potential to significantly reduce the energy needed to heat and cool buildings. Windows provided an introduction to the optical properties of materials. In addition, we revisited the concept of smart materials by exploring options for smart glass, including electrochromic windows. Materials for insulation allowed for further discussion of thermal conductivity of materials. The final unit focused on materials life cycle analysis, with significant time spent on traditional plastics and their biodegradable alternatives. The basics of polymer chemistry were introduced but the discussion was within the context of the end-of-life considerations for plastics. In addition to plastics, students were introduced to materials life cycle analysis approaches and principles used for ecoinformed materials selection. ${ }^{7}$ For each of the four units, students not only learned about materials science concepts, they also were asked to compare various materials that might be used for a particular application and then evaluate the benefits and drawbacks for each material.

Assessment. Because the course was aimed at a broad student audience including environmental studies majors who may not have as much math preparation, problems sets and exams were no longer the only forms of assessment. The focus was on having activities that evaluated conceptual understanding and ability to do quantitative analysis, but not first principles derivations. Shorter problem sets were supplemented with hands-on activities to reinforce concepts. For example, instead of simply discussing elastic and plastic deformation, students were asked to use an apparatus for measuring Young's modulus to explore and report on the mechanical properties of wires made of several different materials. ${ }^{8}$ One activity related to environmental/energy applications of materials had students build and test the performance of a dye-sensitized solar cell. ${ }^{9}$ When exploring smart windows, students deposited and tested the 
behavior of electrochromic Prussian blue thin films. ${ }^{10}$ The course did not include a lab, so all hands-on activities were carried out by students, working in groups of three, during selfscheduled periods outside of class. For each hands-on activity, students were asked to write up their results and connect them to course concepts.

Students also completed a number of writing assignments throughout the course that asked them to either apply what they had learned to new situations or to convey their knowledge to a general audience. These assignments were designed to accommodate varying levels of preparation, with students who had more extensive physics and chemistry background being encouraged to expand their analysis beyond the class readings to include additional sources from the primary literature. For example, one writing assignment asked students to imagine they were serving as a consultant for a philanthropist who wanted to invest in solar energy research; in that context, students had to develop a report prioritizing what areas of solar research they felt merited further investment and why.

Student response. The revised Phys 260 course was taught in 2010-2011, but the composition of students in the class was largely unchanged. Despite direct advertising of the course to ENTS majors via e-mail, not a single ENTS student enrolled. Student response to the revised course was generally positive. The most frequent complaint came from senior physics majors who wanted more mathematically sophisticated derivations and modeling. However, the physics department does offer a solid state physics course on a biennial basis which addresses some materials science topics in a more rigorous fashion. Thus we don't see changing the mathematical rigor of the course as being appropriate, particularly with efforts to keep the course accessible to a broad cross-section of students.

\section{Concluding remarks and future directions}

We have described the revision of an introduction to materials science course housed in the physics department. The original course, aimed at students interested in pursuing MSE, provided a broad overview of materials science. With the revision, course content was pared down to allow for in-depth consideration of a few topics with an environmental focus. The four applications considered were materials for solar energy conversion, smart materials for energy efficiency, materials for green buildings, and materials life cycle analysis. Despite the desire to make the course appealing to students from a variety of majors, the students in the revised course were still primarily physics and chemistry majors, with no ENTS majors enrolling in the revised course. Discussion with students indicated that ENTS majors were uncertain whether the course material would contain sufficient environmental emphasis to be of interest. In addition, some ENTS students were concerned that their lack of background in the physical sciences would be an impediment to being successful in the course. In order to address these concerns, going forward the course will be listed in the environmental studies department (ENTS 262 Materials Science, Energy, and the Environment) instead of in the physics department. The course content has been further revised to reflect the emphasis on energy and environmental applications for materials science, with the most significant change being a significant reduction of the first unit on mechanical properties of materials. In addition, in order to provide further opportunities to explore not just the materials science aspects of particular issues, but also the broader societal 
implications, the course will focus on just three of the four applied topics: materials for solar energy conversion, materials for energy efficient buildings, and materials life cycle analysis.

ENTS 262 Materials Science, Energy, and the Environment will be taught for the first time in the winter of 2013. Preliminary enrollment numbers suggest the change has succeeded in attracting students with a more diverse range of interests, including ENTS majors. In addition, the physics department has agreed that the revised course will continue to meet the department's applied physics requirement for the major so the course enrollment still includes a large number of physics majors. Thus the challenge will remain to offer a course that engages students with a variety of backgrounds in the physical sciences. However, the addition of environmental studies majors should provide more diverse perspectives in classroom discussions and projects.

\section{References}

${ }^{1}$ The original course used the textbook by W.D. Callister, Materials science and engineering: an introduction, (John Wiley \& Sons, USA, 2003), which is a widely used textbook for teaching introductory materials science in materials science and engineering departments.

${ }^{2}$ Changing the Conversation: Messages for Improving Public Understanding of Engineering, National Academy of Engineering, Washington, DC, (2008).

${ }^{3}$ Due to the change in course structure, the revised course used the textbook by R. E. Hummel, Understanding Materials Science: History, Properties, Applications, (Spring-Verlag, NY, 2004), which begins with a focus on the mechanical properties of materials and emphasizes the impact of materials development on the historical development of civilizations.

${ }^{4}$ G.M. Novak, E.T. Patterson, A.D. Gavrin, W. Christian, Just-in-Time Teaching: Blending Active Learning with Web Technology, (Prentice Hall, Upper Saddle River, NJ, 1999).

${ }^{5}$ The starting point we used for students interested in getting an overview of some of the research was Materials Research Society Bulletin Vol 33. No 4 Harnessing Materials for Energy (2008). Now the Materials Research Society has put together an edited collection of essays that can serve as an excellent resource on this topic for students with more advanced scientific backgrounds: D.S. Ginley and D. Cahen,ed., Fundamentals of Materials for Energy and Environmental Sustainability, (Cambridge University Press, UK, 2011).

${ }^{6}$ http://www.colorado.edu/physics/EducationIssues/ModernPhysics/

${ }^{7}$ The supplemental text used for this portion of the course was M.F. Ashby, Materials and the Environment: Ecoinformed materials choice (Butterworth-Heinemann, Waltham, MA, 2009).

${ }^{8}$ A. Niculescu, R. Shumaker, “Apparatus for measuring Young’s modulus,” The Physics Teacher 41, 364 (September 2003).

${ }^{9}$ G.P. Smestad, “Education and solar conversion: Demonstrating electron transfer,” Solar Energy Materials and Solar Cells 55, 157 (1998).

${ }^{10}$ http://education.mrsec.wisc.edu/nanolab/prussianblue/index.html 